\title{
Editorial: Mechanisms of Communication and Recognition in Social Evolution
}

\author{
Mark A. Elgar ${ }^{1 *}$ and Christina Riehl ${ }^{2}$ \\ 'School of BioSciences, University of Melbourne, Melbourne, VIC, Australia, ${ }^{2}$ Department of Ecology and Evolutionary \\ Biology, Princeton University, Princeton, NJ, United States
}

Keywords: social behavior, kin recognition, nestmate recognition, animal signaling, phenotype matching, alarm calls, begging signals, social information

\section{Editorial on the Research Topic}

\section{Mechanisms of Communication and Recognition in Social Evolution}

\section{INTRODUCTION}

The ability to recognize individuals or classes of individuals and to communicate with fellow group members is crucial for the evolution of complex social behavior. At a minimum, cooperating individuals must convey information that identifies themselves as appropriate partners, and collective behavior requires group members to communicate and synchronize their actions. But how do mechanisms of recognition and communication co-evolve with social behavior, and how do similar signaling abilities arise across animal lineages with vastly different sensory systems and cognitive capacities?

While the mechanisms and sensory modalities of communication have been investigated across diverse animal social systems, progress is uneven across both contexts, and taxonomic divides (Elgar, 2015). Perhaps more significantly, these studies are rarely synthesized to seek common patterns across taxa (but see Rubenstein and Abbot, 2017). The Research Topic Mechanisms of Communication and Recognition in Social Evolution takes stock of our current understanding of the proximate mechanisms, selective pressures, and constraints that shape the diversity of communication and recognition systems of social animals, with the intention of inspiring future research directions.

Social animals use signals to communicate information, and an extraordinary diversity of signals have evolved across a range of sensory modalities. Signals may be interpreted in different ways by different individuals or classes of individuals (Stevens, 2013; Wyatt, 2014), and Tumulty and Sheehan argue that understanding the evolution of that diversity requires a shift in the way we think about signals, from considering a single, average receiver to a population of receivers with diverse experiences and motivations. For example, a signal of colony identity may confirm colony membership for some receivers, familiar neighbors for others, and strangers for yet others. Accordingly, Tumulty and Sheehan predict that selection should favor greater uniformity in signals with high receiver agreement, such as for sex recognition, and favor greater signal diversity and flexible learning by receivers for signals where there is low receiver agreement, such as neighbor recognition. The latter prediction suggests that collective learning plays a significant role in determining the level of aggression directed toward non-nestmate neighbors and non-neighbors (e.g., Gill et al., 2012). 


\section{IDENTIFYING APPROPRIATE PARTNERS}

A common feature of social insects and cooperatively breeding vertebrates is their ability to distinguish between nest- or group affiliates and others, including conspecifics, thereby ensuring that the fruits of cooperative behavior benefit the appropriate recipients (Leonhardt et al., 2016). Nevertheless, the nature and focus of research on this topic varies widely along taxonomic lines. Studies of social insects have focused on the nature of the colony identification signal (typically a cocktail of cuticular lipids and hydrocarbons); the genetic and/or environmental source of between-colony variation in that cocktail; and, most vexingly, the mechanism that allows workers to determine whether the chemical profile of an encountered individual differs from their profile (e.g., Rossi and Derégnaucourt, 2020). In contrast, studies of cooperatively breeding birds have primarily focused on the role of acoustic signals in kin recognition in small family groups (Leedale et al.). Comparatively little is known about group-level or kin recognition cues in social mammals, but the available evidence suggests that olfactory signals, including those produced by the major histocompatibility complex (MHC), play a far more important role in mammals than they do in birds (Brennan and Kendrick, 2006).

Perhaps the most notable difference between social insects and cooperatively breeding vertebrates is that group-level recognition cues appear to be the exception rather than the rule in most vertebrate societies. Although learned group-level vocalizations have been experimentally demonstrated in several cooperatively breeding birds (Sharp et al., 2005; Cockburn et al., 2017) and some mammals (Knörnschild et al., 2012), the capacity to distinguish intruders from group members appears to rely primarily on individual recognition, perhaps because the typically smaller group size allows individual familiarity to play a greater role in recognizing intruders (Riehl and Stern, 2015; Leedale et al.).

Group size may also influence the sensory modality of recognition systems in social insects. It is widely understood that olfaction is the primary sensory modality underpinning nestmate recognition in social insects, a conclusion that may be informed by a taxonomic bias toward ants and termites. However, some social insects, including wasps that form relatively small colonies, use visual cues to distinguish between nestmates and nonnestmates (Hunt and Toth, 2017). Clever experiments reported in Cini et al. reveal an increasing reliance on chemical cues by the wasp Polistes dominula with increasing colony size: visual cues are used to distinguish familiar individuals, such as nestmates, from others during the early stages of colony development when colonies are typically small, while older and larger colonies increasingly rely on chemical signals. With larger numbers of individuals within a colony, the capacity to recognize individuals may be impossible, and so workers must recognize classes of individuals. Visual cues may be more efficient for rapidly recognizing a relatively small number of individuals (see Baracchi et al., 2015), since they can accommodate considerable diversity of variants on a common theme (such as facial color pattern), but the extent of this variation may be impractical for chemical signals, where different chemical mixtures may require different receptors. As colony size increases, chemical signals may be more efficient because within-class variation, representing different colony membership or different tasks within a colony, may be less than betweenclass variation.

Much of the focus of the role of communication in maintaining group cohesion centers around identifying and excluding individuals that are not members of the nest or colony. However, many forms of social behavior require communication to recruit individuals to join groups. Individuals may attract the attention of other group members using food and alarm signals, and individuals of species that form open membership groups may similarly recruit others to a group, if the benefits of increasing group size outweigh the costs (e.g., Elgar, 1986). The capacity to attract conspecifics is critical in the formation of shoals of fish, but these signals may be directed at particular classes of individuals. In an extensive review, Ward et al. document the remarkable preferences of shoal forming fish for particular kinds of associates, based on sex, size, health, relatedness, familiarity, and even color patterns in species with color pattern polymorphism. The benefits to individuals that exercise these preferences for shoal mates have been explored extensively, and fish use a variety of sensory modalities and recognition templates to enable these preferences. The most common recognition template appears to be phenotype matching (see Hauber and Sherman, 2001), which is thought to be learned.

The nests of social insects offer rich pickings for natural enemies, which include a large and taxonomically diverse number of "social parasites" that live within the nest and, in some species, feed on the vulnerable larvae. Given the impressive capacity of social insects to distinguish between nestmates and others, how do these unwanted guests remain safely within the nest of their host? One mechanism is chemical mimicry, where the cuticular chemistry of the guests mimic that of the host, thereby allowing the guests unfettered access to the nest (e.g., Allan et al., 2002). Alternatively, unwanted guests could avoid detection through "chemical insignificance," a term coined by Lenoir et al. (2001) that emphasizes an absence of chemical recognition cues similar to that of just-eclosed callow workers. In their review, Lorenzi and d'Ettorre identify two other ways in which unwanted guests might remain effectively "chemically" insignificant: by ensuring the concentration of cuticular hydrocarbons remains below the discriminator's detection threshold and/or by producing hydrocarbons that are not recognized as cues by the hosts. Rather than erecting new terminology to describe these mechanisms, Lorenzi and d'Ettorre argue it would be more convenient to expand the definition of "chemical insignificance" to incorporate these additional ways in which intruders remain undetected. It is not known whether selection has favored the elimination of detectable odors in these systems, which raises the broader value of using definitions that do not necessarily imply a function. Such "neutral" nomenclature would not exclude consideration of non-adaptive or nonfunctional explanations, an approach that is emerging for other sensory modalities. 


\section{SOCIAL INFORMATION AND APPROPRIATE TASKS}

Engaging in social activities self-evidently requires communication, typically in the form of signals that have evolved to alter the behavior of the recipient. This signaling might reflect changes in the environment, e.g., the appearance of predators, or changes in the requirements of the signaller, e.g., by begging for food.

Alarm calls are a conspicuous form of communication in many vertebrates, where individuals vocalize in the presence of predators (Caro, 2005). An ongoing challenge of this research is to demonstrate whether these calls reflect the state of the signaller, or represent functionally referential signals, where the calls refer to particular predators (e.g., Gill and Bierema, 2013; Townsend and Manser, 2013). Nouri and Blumstein show that the nature of alarm calls in marmots are affected by infection with a coccid parasite (although not with infection of several other parasites). Specifically, the calls of infected individuals have higher Wiener entropy values (reflecting higher "randomness" in the sound waves), and so are less precise than those of uninfected individuals. The functional significance, if any, of this variation in alarm calls is unclear because it is not known if or how potential receivers respond. Alarm calls may be used as cues by predators to determine whether the signaller is healthy and thus less easily captured; if so, more vulnerable, infected signalers would be expected to desist or reduce alarm calling, since it increases their risk of predation. On the other hand, alarm calls may also alert conspecifics of potential danger, so the precision of the alarm signal could theoretically provide additional information about its reliability to these receivers. For example, the contact calls of juvenile marmosets have higher Wiener entropy values than those of adults (Takahashi et al., 2017), so the Wiener entropy value of alarm calls could provide information about their reliability if it varies with signaller age.

In social insects, colony efficiency is thought to be improved by workers engaging in specific tasks. The allocation of workers to particular tasks is self-organized, where the collective evaluation of the relative abundance of workers engaged in different activities within the nest relies on individuals being able to recognize the task of nestmates. While several studies confirm that this information is conveyed through chemical signals, typically cuticular hydrocarbons, the nature of these differences is difficult to elucidate because the chemical profile is derived from an extraction of the entire body of the insect. Wang et al. (2016) discovered that the greatest between-colony differences in cuticular hydrocarbons were located on the antennae of meat ants Iridomyrmex purpureus, and that workers pay most attention to the antennae of non-nestmates. Following this insight, Wang et al. reveal that task identification signals are located primarily on the legs of workers. Their experiments provide two important insights: that task identification signals are not colony specific; and that cuticular based signaling in social insects is location specific. It remains to be seen whether social insects in general perceive cuticular chemical signals by targeting particular body parts, but this result highlights an important issue-chemical analysis of the cuticular chemical signature of whole-body extracts may be misleading because they can conflate many different signals.

While the honesty of begging signals figures significantly in studies of breeding birds (Mock et al., 2011), little is known about begging honesty in cooperatively breeding species (e.g., MacLeod and Brouwer, 2018), and analyses of provisioning in social insects have largely focused on worker behavior, rather than how larvae signal their nutritional requirements (but see $\mathrm{He}$ et al., 2016; Pepiciello et al., 2018). Peignier et al. provide one of the first studies to investigate how genetic factors influence begging by the larvae of ants. In species with higher levels of genetic relatedness, food-deprived ant larvae tend to beg more than their nourished counterparts, but this effect was not apparent in species with low within-colony relatedness, where larvae either did not adjust their behavior or begged more when they were nourished. This study provides a fascinating parallel with recent comparative analyses in birds, which have struggled to find strong correlations between begging honesty and genetic relatedness within the extended family (e.g., Caro et al., 2016; Bebbington and Kingma, 2017). Although begging signals are often unreliable when nest-mates are wholly unrelated to each other-for example, when broods are parasitized by heterospecific nest parasitesthe honesty of begging signals does not predictably covary with fine-scale genetic relatedness, probably because it is confounded by variation in the intensity of competition between nest-mates (Bebbington and Kingma, 2017). Peignier et al. nicely circumvent this problem by experimentally manipulating food availability, effectively controlling the extent of competition between larvae. While this study should encourage investigations of social insect larvae as active players in colony life (see also Schultner et al., 2014), it also highlights the importance of considering the intended signal receivers. Varying signaling effort is unlikely to be effective if it does not exact a response: workers did not move significantly more toward the odor of starved larvae than toward the odor of non-starved larvae, perhaps reflecting the lack of differences in cuticular hydrocarbons, alkanes, methylated alkanes, and alkenes between these two groups of larvae. This result contrasts with bumblebees, which reacted more strongly to the odors of food deprived larvae (den Boer and Duchateau, 2006).

The mechanisms of kin discrimination in social birds are thought to involve external cues, often learned during development, which can be used by a receiver as a proxy for genetic relatedness (Leedale et al.). Receivers may infer kinship through familiarity and/or phenotype matching; "genetic" kin recognition is theoretically unlikely and rarely demonstrated empirically (Riehl and Strong, 2015). While familiarity is widely understood to be the primary mechanism that allows kin discrimination, Leedale et al. point out that we have an incomplete understanding of how familiarity allows individuals to recognize kin: what cues are used, what is the sensitive period for association, and how can we distinguish between familiarity and phenotype matching? While vocal cues seem to be the most likely sensory modality, this may reflect an historical research 
bias, and visual and olfactory cues cannot be ruled out. It is also important to ask whether finely tuned discrimination is adaptive in the context of the behavioral response. When the receiver's decision is binary-e.g., to feed or not to feed a begging nestmate-the basis of the decision may reflect a threshold rather than quantitative evaluation of the degree of relatedness. Finally, the capacity to discriminate is unlikely where the potential recipients do not vary in relatedness.

Individuals in social groups can be both the source and recipients of information transmitted within the group, which can include the nature and location of potential food, competitors and predators. Individuals may also pay attention to the mating preferences of others within the group, and tend to copy those preferences, a behavior that was first reported roughly 50 years ago (Wiley, 1973; Lill, 1974; Dugatkin, 1992), and subsequently investigated across a broad range of taxa. The metaanalysis by Jones and DuVal reveals that females, and especially inexperienced females, pay attention to the mating behavior of other females, typically preferring to mate with a male if he had been "endorsed" by other females through positive social information. Indeed, observer females were around six times more likely to mate with a generally unpreferred male, if they had seen that male mate with another female. Interestingly, the testing condition (wild or captive) had the strongest impact on mate-choice copying, with females in free-living populations more likely to copy the mate choice of others than females in captive populations. Jones and DuVal identify several possible explanations, but perhaps the most compelling is that females in natural populations are making more genuine choices than those elicited in the typically dichotomous laboratory choices. Clearly, this begs the question of what is the nature of the information observing females acquire, beyond the act of mating-are they learning something about male quality? More generally, it would be interesting to ask whether individuals use social information to make decisions in other contexts, such as responding to rivals and competitors (e.g., Aquiloni et al., 2008; Tibbetts et al., 2020).

\section{PROSPECTS}

The nine papers included in this Research Topic illustrate and build on many of the major advances in the field of social animal communication over the past several decades, from conceptual developments in signal honesty and evolution (e.g., Peignier et al.) to the technical innovations that allow human researchers to eavesdrop on the chemosensory modalities of their study animals (e.g., Wang et al.; Lorenzi and d'Ettore). There is an increasing realization that common selective pressures and constraints may underlie the evolution of communication mechanisms in seemingly disparate contexts (for example, mate choice, kin discrimination, and intraspecific competition; Jones and DuVal; Tumulty and Sheehan; Leedale et al.) and that seeking parallels across contexts and taxa may yield the greatest insights into these evolutionary processes. However, this Research Topic also highlights the many gaps remaining in our knowledge and raises new challenges for future research.
Many questions remain about the cognitive mechanisms that enable recognition cues to be learned. For example, phenotype matching is thought to be widely used to identify membership of particular classes of individuals, including potential mating partners (e.g., Kopp et al., 2018); neighbors (Crepy and Casal, 2015, but see Till-Bottraud and de Villemereuil, 2016); offspring (e.g., Yang et al., 2019); and social groups (Ward et al.). Phenotype matching is especially important for social species, to direct cooperative behavior toward particular individuals (typically group or colony mates; Leedale et al.; Rossi and Derégnaucourt, 2020) or to detect extra-group parasites (Shizuka and Lyon, 2010). Phenotype matching occurs when the individual references its own traits, often referred to as a template, against that of another individual, and responds accordingly (Hauber and Sherman, 2001; Mateo, 2004). The template comprises signals (or cues) that may include odors, sounds or color patterns. While there is evidence for such templates (e.g., Rossi and Derégnaucourt, 2020), very little is known about how individuals learn a self-referencing template. For vertebrates, the challenge is to distinguish whether recognition results from familiarity or phenotype matching, to determine the extent to which self-referential templates might be genetically determined, and to identify developmentally sensitive periods in which such templates might be learned (Leedale et al.). For social insects that rely primarily on chemical cues found on the insect cuticle (but see Cini et al.), the challenge lies in accounting for the many factors that may change the nature of these chemicals, including diet, ontogeny, and task (Henneken et al., 2017; Otte et al., 2018; Wang et al.); and that a complete absence of cues is an effective mechanism of by-passing this recognition mechanism (Lorenzi and d'Ettorre). Perhaps familiarity plays an important role in recognition cues in social insects (e.g., Gill et al., 2012).

Another challenge is to understand how the physiology and morphology of sensory systems of receivers have coevolved with social signals. In social mammals, neurological structures associated with olfactory learning and recognition of individuals have coevolved with the expression of odorant molecules, and chemo signals that likely originated in the context of mating and parental care have been co-opted for use in cooperative behaviors (for example, in kin recognition; Brennan and Kendrick, 2006). More recent evidence in social insects suggests similar coevolutionary patterns between social behavior and receptor organ morphology (Wittwer et al., 2017), and evolutionary links between social behavior, signals, learning, and perception may emerge in other systems and species (Miller et al., 2020). Vocal repertoire size in primates is correlated with group size (McComb and Semple, 2005), but in birds has increased with the evolution of cooperative breeding, but not group size or group stability (Leighton, 2017). The associated neurological requirements for processing this information have led to hypothesized links between social complexity and brain size, with mixed empirical support across taxa (Godfrey and Gronenberg, 2019). Does social complexity, and its attendant requirements for sophisticated communication and individual recognition, drive signal evolution? Or do pre-existing coevolved sensory systems 
between signalers and receivers allow societies to increase in complexity (Freeberg et al., 2012)? While it is self-evident that communication underpins social behavior, unpicking these details continues to offer intriguing research opportunities.

\section{AUTHOR CONTRIBUTIONS}

ME and CR conceived, wrote, and edited the manuscript, and approved the submitted version.

\section{REFERENCES}

Allan, R. A., Capon, R. J., Brown, V., and Elgar, M. A. (2002). Mimicry of host cuticular hydrocarbons by salticid spider Cosmophasis bitaeniata that preys on larvae of tree ants Oecophylla smaragdina. J. Chem. Ecol. 28, 835-848. doi: 10.1023/A:1015249012493

Aquiloni, L., Buřrič, M., and Gherardi, F. (2008). Crayfish females eavesdrop on fighting males before choosing the dominant mate. Current Biol. 18, R462R463. doi: 10.1016/j.cub.2008.04.006

Baracchi, D., Petrocelli, I., Chittka, L., Ricciardi, G., and Turillazzi, S. (2015). Speed and accuracy in nest-mate recognition: a hover wasp prioritizes face recognition over colony odour cues to minimize intrusion by outsiders. Proc. R. Soc. B 282, 20142750. doi: 10.1098/rspb.2014.2750

Bebbington, K., and Kingma, S. A. (2017). No evidence that kin selection increases the honesty of begging signals in birds. Evol. Lett. 1, 132-137. doi: $10.1002 /$ evl3.18

Brennan, P. A., and Kendrick, K. M. (2006). Mammalian social odours: attraction and individual recognition. Phil. Trans. R. Soc B 361, 2061-2078. doi: $10.1098 /$ rstb.2006.1931

Caro, S. M., West, S. A., and Griffin, A. S. (2016). Sibling conflict and dishonest signaling in birds. Proc. Natl. Acad. Sci. U.S.A. 113, 13803-13808. doi: $10.1073 /$ pnas. 1606378113

Caro, T. (2005). Antipredator Defenses in Birds and Mammals. Chicago: Chicago University Press.

Cockburn, A., Hatchwell, B. J., and Koenig, W. D. (2017). "Sociality in birds”, in Comparative Social Evolution, eds D. R. Rubenstein and P. Abbot (Cambridge: Cambridge University Press), 320-353. doi: 10.1017/9781107338319.012

Crepy, M. A., and Casal, J. J. (2015). Photoreceptor-mediated kin recognition in plants. New Phytol. 205, 329-338. doi: 10.1111/nph.13040

den Boer, S. P. A., and Duchateau, M. J. H. M. (2006). A larval hunger signal in the bumblebee Bombus terrestris. Insect Soc. 53, 369-373. doi: 10.1007/s00040-006-0883-8

Dugatkin, L. A. (1992). Sexual selection and imitation: females copy the mate choice of others. Am. Nat. 139, 1384-1389. doi: 10.1086/285392

Elgar, M. A. (1986). House sparrows establish foraging flocks by giving chirrup calls if the resources are divisible. Anim. Behav. 34, 169-174. doi: 10.1016/0003-3472(86)90020-5

Elgar, M. A. (2015). Integrating insights across diverse taxa: challenges for understanding social evolution. Front. Ecol. Evol. 3:124. doi: $10.3389 /$ fevo.2015.00124

Freeberg, T. M., Dunbar, R. I., and Ord, T. J. (2012). Social complexity as a proximate and ultimate factor in communicative complexity. Phil. Trans. Roy. Soc. B 367, 1785-1801. doi: 10.1098/rstb.2011.0213

Gill, K. P., van Wilgenburg, E., Taylor, P., and Elgar, M. A. (2012). Collective retention and transmission of chemical signals in a social insect. Naturwissenschaften 99, 245-248 doi: 10.1007/s00114-0120891-7

Gill, S. A., and Bierema, A. M.-K. (2013). On the meaning of alarm calls: a review of functional reference in avian alarm calling. Ethology 119, 449-461. doi: $10.1111 /$ eth.12097

Godfrey, R. K., and Gronenberg, W. (2019). Brain evolution in social insects: advocating for the comparative approach. J. Comp. Phys. A. 205, 13-32. doi: 10.1007/s00359-019-01315-7

\section{ACKNOWLEDGMENTS}

We thank the authors of the nine papers for their timely and thought-provoking contributions to this Research Topic; the scientific committee of the Second Joint Conference on Evolutionary Biology (Montpellier 2018) for including our symposium Mechanisms of communication and recognition in social evolution, which formed the basis of this Research Topic; and the helpful comments of the Review Editor.

Hauber, M. E., and Sherman, P. W. (2001). Self-referent phenotype matching: theoretical considerations and empirical evidence. Trends Neurosci. 24, 609-616. doi: 10.1016/S0166-2236(00)01916-0

He, X. J., Zhang, X. C., Jiang, W. J., Barron, A. B., Zhang, J. H., and Zeng, Z. J. (2016). Starving honey bee (Apis mellifera) larvae signal pheromonally to worker bees. Scientific Rep. 6, 1-9. doi: 10.1038/srep22359

Henneken, J., Jones, T. M., and Elgar, M. A. (2017) Diet-mediated pheromones and signature mixtures can enforce signal reliability. Front. Ecol. Evol. 4:145. doi: $10.3389 /$ fevo.2016.00145

Hunt, J. H., and Toth, A. L. (2017). "Sociality in wasps," in Comparative Social Evolution, eds D. R. Rubenstein and P. Abbot (Cambridge: Cambridge University Press), 84-123. doi: 10.1017/9781107338319.005

Knörnschild, M., Nagy, M., Metz, M., Mayer, F., and von Helversen, O. (2012). Learned vocal group signatures in the polygynous bat Saccopteryx bilineata. Anim. Behav. 84, 761-769. doi: 10.1016/j.anbehav.2012.06.029

Kopp, M., Servedio, M. R., Mendelson, T. C., Safran, R. J., Rodríguez, R. L., Hauber, M. E., et al. (2018). Mechanisms of assortative mating in speciation with gene flow: Connecting theory and empirical research. Am. Nat. 191, 1-20. doi: $10.1086 / 694889$

Leighton, G. M. (2017). Cooperative breeding influences the number and type of vocalizations in avian lineages. Proc. R. Soc. B. 284, 20171508 doi: 10.1098/rspb.2017.1508

Lenoir, A., d'Ettorre, P., Errard, C., and Hefetz, A. (2001). Chemical ecology and social parasitism in ants. Ann. Rev. Entomol. 46, 573-599. doi: 10.1146/annurev.ento.46.1

Leonhardt, S. D., Menzel, F., Nehring, V., and Schmitt, T. (2016). Ecology and evolution of communication in social insects. Cell 164, 1277-1287. doi: $10.1016 /$ j.cell.2016.01.035

Lill, A. (1974). Sexual behavior of the lek-forming white-bearded manakin (Manacus manacus trinitatis Hartert). Z. Tierpsychol. 36, 1-36. doi: 10.1111/j.1439-0310.1974.tb02126.x

MacLeod, K. J., and Brouwer, L. (2018). Social context-dependent provisioning rules in red-winged fairy-wrens do not vary with signals of increased chick need. Anim. Behav. 143, 105-111. doi: 10.1016/j.anbehav.2018.07.010

Mateo, J. M. (2004). Recognition systems and biological organization: the perception component of social recognition. Ann. Zool. Fennici 41, 729-745. Available online at: https://www.jstor.org/stable/23736140

McComb, K., and Semple, S. (2005). Coevolution of vocal communication and sociality in primates. Biol. Lett. 1, 381-385. doi: 10.1098/rsbl.2005.0366

Miller, S. E., Sheehan, M. J., and Reeve, H. K. (2020). Coevolution of cognitive abilities and identity signals in individual recognition systems. Phil. Trans. $R$. Soc B 375:20190467. doi: 10.1098/rstb.2019.0467

Mock, D. W., Dugas, M. B., and Strickler, S. A. (2011). Honest begging: expanding from Signal of Need. Behav. Ecol., 22, 909-917. doi: 10.1093/beheco/arr091

Otte, T., Hilker, M., and Geiselhardt, S. (2018). Phenotypic plasticity of cuticular hydrocarbon profiles in insects. J. Chem. Ecol. 44, 235-247. doi: 10.1007/s10886-018-0934-4

Pepiciello, I., Cini, A., Nieri, R., Mazzoni, V., and Cervo, R. (2018). Adult-larval vibrational communication in paper wasps: the role of abdominal wagging in Polistes dominula. J. Exp. Biol. 221:186247. doi: 10.1242/jeb.186247

Riehl, C., and Stern, C. A. (2015). How cooperatively breeding birds identify relatives and avoid incest: new insights into dispersal and kin recognition. BioEssays 37, 1303-1308. doi: 10.1002/bies.201500120 
Rossi, N., and Derégnaucourt, S. (2020). Mechanisms of recognition in birds and social Hymenoptera: from detection to information processing. Phil. Trans. R. Soc. B 375:20190483. doi: 10.1098/rstb. 2019.0483

Rubenstein, D. R., and Abbot, P. (2017). "Social synthesis: opportunities for comparative social evolution," in Comparative Social Evolution, eds D. R. Rubenstein and P. Abbot (Cambridge: Cambridge University Press), 427-452. doi: $10.1017 / 9781107338319.015$

Schultner, E., Gardner, A., Karhunen, M., and Helanter,ä, H. (2014). Ant larvae as players in social conflict: relatedness and individual identity mediate cannibalism intensity. Am. Nat. 184, E161-E174. doi: 10.1086/ 678459

Sharp, S. P., McGowan, A., Wood, M. J., and Hatchwell, B. J. (2005). Learned kin recognition cues in a social bird. Nature 434, 1127-1130. doi: 10.1038 /nature 03522

Shizuka, D., and Lyon, B. E. (2010). Coots use hatch order to learn to recognize and reject conspecific brood parasitic chicks. Nature 463, 223-226. doi: 10.1038 /nature 08655

Stevens, M. (2013). Sensory Ecology, Behaviour, and Evolution. Oxford: Oxford University Press. doi: 10.1093/acprof:oso/9780199601776.001.0001

Takahashi, D. Y., Liao, L. A., and Ghazanfar, A. A. (2017). Vocal learning via social reinforcement by infant marmoset monkeys. Curr. Biol. 27, 1844-1852. doi: 10.1016/j.cub.2017.05.004

Tibbetts, E. A., Wong, E., and Bonello, S. (2020). Wasps use social eavesdropping to learn about individual rivals. Curr. Biol. 30, 3007-3010. doi: 10.1016/j.cub.2020.05.053

Till-Bottraud, I., and de Villemereuil, P. (2016). Kin recognition or phenotype matching? N. Phytol. 209, 13-14. doi: 10.1111/nph.13554
Townsend, S. W., and Manser, M. B. (2013). Functionally referential communication in mammals: the past, present and the future. Ethology 119, 1-11. doi: 10.1111/eth.12015

Wang, Q., Goodger, J. Q. D., Woodrow, I. E., and Elgar, M. A. (2016). Locationspecific cuticular hydrocarbon signals in a social insect. Proc. R. Soc. B 283:20160310. doi: 10.1098/rspb.2016.0310

Wiley, R. H. (1973). Territoriality and non-random mating in sage grouse, Centrocercus urophasianus. Anim. Behav. Monogr. 6, 85-169. doi: 10.1016/0003-3472(73)90004-3

Wittwer, B., Hefetz, A., Simon, T., Murphy, L. E. K., Elgar, M. A., Pierce, N. E., et al. (2017). Solitary bees reduce investment in communication compared with their social relatives. Proc Nat. Acad. Sci. USA. 114, 6569-6574. doi: $10.1073 /$ pnas.1620780114

Wyatt, T. D. (2014). Pheromones and Animal Behaviour: Chemical Signals and Signatures (2nd ed). Cambridge: Cambridge University Press.

Yang, C., Liang, W., and Møller, A. P. (2019). Egg retrieval versus egg rejection in cuckoo hosts. Phil. Trans. R. Soc. B 374:20180200. doi: 10.1098/rstb.2018.0200

Conflict of Interest: The authors declare that the research was conducted in the absence of any commercial or financial relationships that could be construed as a potential conflict of interest.

Copyright $(02021$ Elgar and Riehl. This is an open-access article distributed under the terms of the Creative Commons Attribution License (CC BY). The use, distribution or reproduction in other forums is permitted, provided the original author $(s)$ and the copyright owner(s) are credited and that the original publication in this journal is cited, in accordance with accepted academic practice. No use, distribution or reproduction is permitted which does not comply with these terms. 